\title{
Anthony Busuttil, Jean W. Keeling (eds): Paediatric forensic medicine and pathology
}

\author{
Hodder Arnold (2009), pp. 504, hardback ISBN-13 9780340731574
}

\author{
Jonathon Herbst
}

Accepted: 14 May 2009/Published online: 4 August 2009

(C) Humana Press 2009

Pediatric forensic cases can often be complex requiring input from several different sources of expertise. These cases require a complete and comprehensive evaluation. Busuttil and Keeling have done a masterful job in assembling a book that serves as a resource for just these types of cases. The editors have gone to great lengths to garner expertise from a variety of sources including experts in hematology, pediatric radiology and others. The finished product of 24 chapters covers the entire spectrum of pediatric forensics.

The chapters are comprehensive and in-depth. The authors' styles are well packaged and broken up into digestible sections. Topics can be easily located and navigated by the user-friendly table of contents and index. The unifying trait, which makes this text exemplary, is the use of pictures and charts. Pictures are used to illustrate and reinforce textural concepts, and they are all of the highest colour quality and vibrancy, with findings well centred and easily identified in the pictures. Charts provide essential tips and information without being excessively wordy. The pictures throughout the text are pertinent and there do not appear to be any included unnecessarily. Chapters are well researched with the latest references augmenting the text, and fillers of pedagogic statistics avoided. The editors have done well by keeping the text readable with good tempo and pace. Two appendices provide a useful resource of charts, diagrams, and tables.
This text caters to the uniqueness of the field, e.g., there are sections that review agricultural injuries, child cyclists and nearly every other conceivable form, or method, which may result in pediatric injury or death. It was especially refreshing to find chapters focused on specific venues such as the chapter on sudden death of children in hospital settings. Topics from this chapter include deaths in the dental chair, therapeutic misadventures, and deaths due to medical devices and procedures. Other chapters are likewise methodical and contain a fresh approach to pertinent topics. For example bath tub drownings are explored in depth to cover scenarios that include accidents, homicide, epilepsy, and euthanasia. Additionally, bath tub drowning and organic pathology is discussed. My only negative comment is that some areas in the overall text contain extensively long numbered lists of facts. These may have been better placed in a tabular format.

Overall I found this text to be well researched and easy to navigate with excellent tables and pictures. It is remarkably applicable to cases one comes across in practice, either in clinical forensics or forensic pathology, and is highly recommended to those in the fields of clinical or forensic pathology, as well as pediatric forensic pathologists and pediatric pathologists. Those in the legal profession who are involved in these cases would also benefit by owning this text.

\footnotetext{
J. Herbst $(\square)$

Forensic Science South Australia, 21 Divett Place,

Adelaide, SA 5000, Australia

e-mail: herbst.jonathon@saugov.sa.gov.au
} 